\title{
Anaplastic (Malignant) Intracranial Meningioma
}

National Cancer Institute

\section{Source}

National Cancer Institute. Anaplastic (Malignant) Intracranial Meningioma. NCI

Thesaurus. Code C27307.

An anaplastic mening ioma that arises within the cranial cavity. 